\title{
Nonlinear Analysis of Ordinary Bridges Crossing Fault-Rupture Zones
}

\author{
Rakesh K. Goel, California Polytechnic State University, San Luis Obispo \\ and Anil K. Chopra, University of California, Berkeley
}

\begin{abstract}
Rooted in structural dynamics theory, three approximate procedures for estimating seismic demands for bridges crossing fault-rupture zones and deforming into their inelastic range are presented: modal pushover analysis (MPA), linear dynamic analysis, and linear static analysis. These procedures estimate the total seismic demand by superposing peak values of quasi-static and dynamic parts. The peak quasi-static demand in all three procedures is computed by nonlinear static analysis of the bridge subjected to peak values of all support displacements applied simultaneously. In the MPA and the linear dynamic analysis procedures, the peak dynamic demand is estimated by nonlinear static (or pushover) analysis and linear static analysis, respectively, for forces corresponding to the most-dominant mode. In the linear static analysis procedure, the peak dynamic demand is estimated by linear static analysis of the bridge due to lateral forces appropriate for bridges crossing fault-rupture zones. The three approximate procedures are shown to provide estimates of seismic demands that are accurate enough to be useful for practical applications. The linear static analysis procedure, which is much simpler than the other two approximate procedures, is recommended for practical analysis of "ordinary" bridges because it eliminates the need for mode shapes and vibration periods of the bridge.
\end{abstract}

\section{Introduction}

The companion paper (Goel and Chopra 2009) developed a response spectrum analysis (RSA) procedure and a linear static analysis procedure for estimating a dynamic part of seismic demands in linearly elastic bridges crossing fault-rupture zones and demonstrated that both these procedures, when combined with the quasi-static demands due to ground offset across the fault, provide estimates of peak responses that are close to the exact results from response history analysis (RHA). However, bridges crossing fault-rupture zones are expected to be deformed beyond their linear elastic range. Therefore, the objective of the investigation reported in this paper is to extend the aforementioned procedures to estimate seismic demands for "ordinary" bridges (defined in the companion paper) deforming into their inelastic range.

The structural systems and modeling approaches considered in this investigation are the same as those in the companion paper (Goel and Chopra 2009) except for modeling of columns. The columns in this investigation are modeled with the nonlinearBeamColumn element in the structural analysis software Open System for Earthquake Engineering Simulation (OpenSees) (McKenna and Fenves 2001). The nonlinearBeamColumn element uses a force-based, distributed-plasticity approach with integration of section behavior over the member length. The section is defined with fibers of confined concrete, unconfined concrete, and steel reinforcing bars. The nonlinear axial-flexural behavior of the element is determined by integration of the nonlinear stress-strain relationships of various fibers across the section, whereas linear behavior is assumed for shear and torsional behavior. The compressive stress-strain behavior of concrete, both confined and unconfined, was modeled with Concrete01 material in OpenSees. The tensile strength of concrete was ignored. Further, concrete was assumed to completely lose strength immediately after the crushing strain. The crushing strain of the unconfined concrete was selected to be equal to 0.004 and that for confined concrete was selected to be that corresponding to the rupture of confining steel using the well-established Mander model (Mander et al. 1988). The stress-strain behavior of steel was modeled with ReinforcingSteel material in OpenSees. Further details of the material models are available in McKenna and Fenves (2001).

For reasons noted in the companion paper ( Goel and Chopra 2009), two extreme shear key cases-without shear keys and with elastic shear keys-were considered in this investigation. Details on shear key behavior and computer modeling are available in Goel and Chopra (2008b). The ground motions considered in this phase of the investigation are the proportional multiple-support excitations described in the companion paper (Goel and Chopra 2009).

Presented first is the theoretical background followed by development of three approximate procedures for analysis of nonlinear bridges crossing fault-rupture zones. Subsequently, accuracy of these procedures is evaluated. Finally, comments on a procedure currently being used by practicing engineers are provided. 

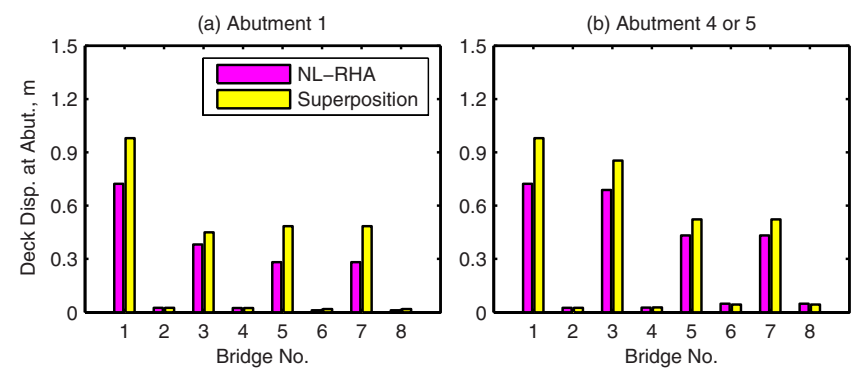

Fig. 1. Comparison of transverse deck displacements at abutments determined by two analyses: exact nonlinear RHA (NL-RHA) and superposition of peak values of nonlinear quasi-static and nonlinear dynamic response. Results are for fault-parallel ground motions associated with a strike-slip fault.

\section{Superposing Quasi-Static and Dynamic Parts of Response}

Inelastic response analysis of bridges subjected to multiplesupport excitation requires a step-by-step solution of equations governing the total displacements $\mathbf{u}^{t}$ of the bridge for ground motions directly imposed on the support degrees of freedom of the system. This procedure, denoted as exact nonlinear RHA, is too onerous for estimating seismic demands for ordinary bridges. With the objective of developing practical procedures, we explore whether an approximate solution based on superposition of the peak values of the quasi-static and dynamic part of the response [Eq. (17) of the companion paper (Goel and Chopra 2009)] provides acceptable estimates for the inelastic seismic demands for bridges. The peak values of quasi-static and dynamic responses, $\mathbf{u}_{o}^{s}$ and $\mathbf{u}_{o}$, are computed by two independent nonlinear analyses of the bridge: (1) $\mathbf{u}_{o}^{s}$ is determined by nonlinear static analysis of the bridge subjected to peak values of ground displacements, $\alpha_{l} u_{g o}$, simultaneously applied at all supports; and (2) $\mathbf{u}_{o}$ is determined by nonlinear dynamic analysis, i.e., solving the equations of motion:

$$
\mathbf{m} \ddot{\mathbf{u}}+\mathbf{c u}+\mathbf{f}_{s}(\mathbf{u}, \dot{\mathbf{u}})=-\mathbf{m}_{\mathrm{eff}} \ddot{u}_{g}(t)
$$

The peak values of seismic demands obtained by this approximate superposition procedure are compared against those from exact nonlinear RHA in Figs. 1 and 2 for eight selected bridges. The presented results indicate that this approximate procedure generally leads to a conservative-but not excessively conservative — estimate of deck displacements at abutments (Fig.
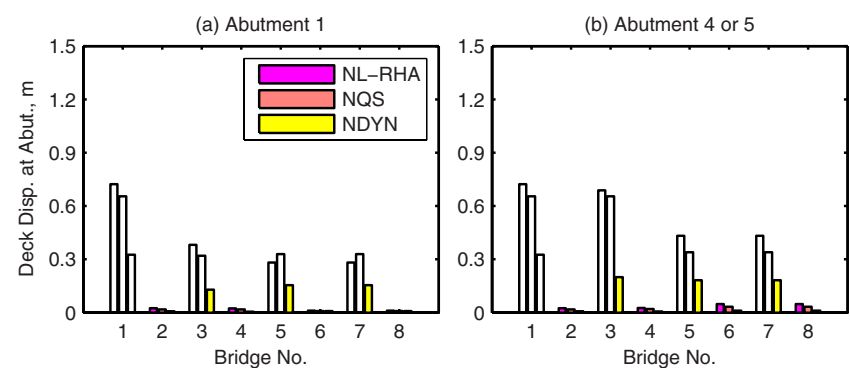

Fig. 3. Comparison of transverse deck displacements at abutments determined by three analyses: nonlinear RHA (NL-RHA), nonlinear quasi-static (NQS), and nonlinear dynamic (NDYN). Results are for fault-parallel ground motions associated with a strike-slip fault.

1) and column drifts (Fig. 2). Exceptions occur for column drift in Bent 2 of Bridges 5 and 7, where the superposition leads to slightly smaller estimate [Fig. 2(a)].

The preceding results indicate that, although superposition of peak quasi-static and dynamic responses determined by two independent nonlinear analyses is not "strictly" valid, this approach provides estimates of seismic demands that are accurate to a useful degree. This is the approach adopted to develop a practical procedure for estimating inelastic seismic demands for bridges.

\section{Is Quasi-Static Solution Adequate?}

Because the displacement offset associated with fault rupture dominates the earthquake excitation, can the structural response be approximated by the quasi-static solution alone? To address this question, the peak values of the total response are presented in Figs. 3 and 4, together with the peak values of the quasi-static and dynamic parts of the response. These results indicate that the peak values of the total deck displacement at bridge abutments may be estimated from nonlinear quasi-static analysis alone (Fig. 3 ); however, the quasi-static response alone is inadequate in estimating column drifts in bridges without shear keys [see Bridges 1, 3, 5, and 7 in Fig. 4(a); Bridges 1 and 3 in Fig. 4(b); and Bridges 5 and 7 in Fig. 4(c)].

\section{Estimation of Peak Response}

Approximate procedures proposed herein are based on superposing quasi-static and the dynamic parts of the response, an ap- (a) Bent 2

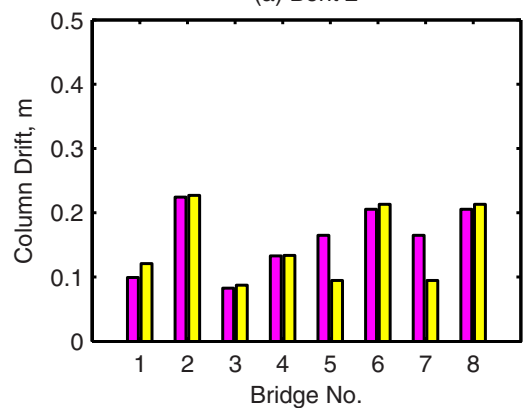

(b) Bent 3

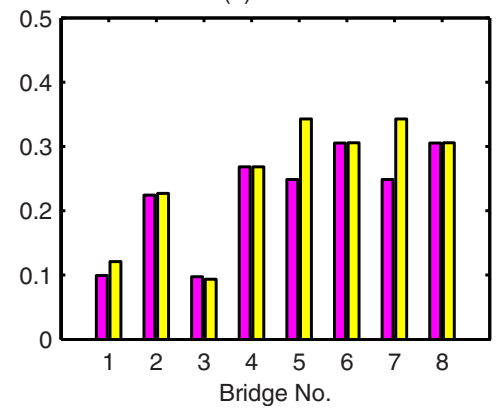

(c) Bent 4

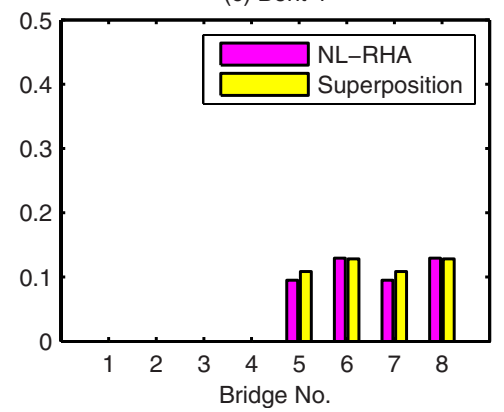

Fig. 2. Comparison of transverse column drifts determined by two analyses: exact nonlinear RHA (NL-RHA) and superposition of peak values of nonlinear quasi-static and nonlinear dynamic response. Results are for fault-parallel ground motions associated with a strike-slip fault. 
(a) Bent 2

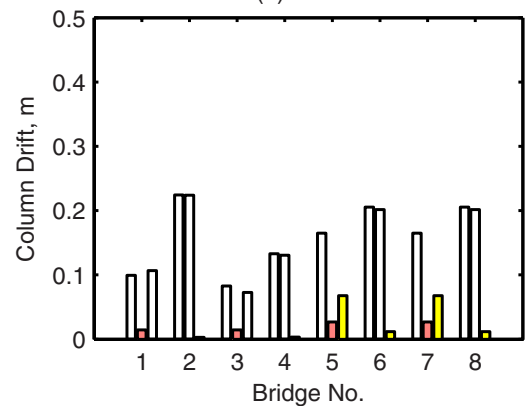

(b) Bent 3

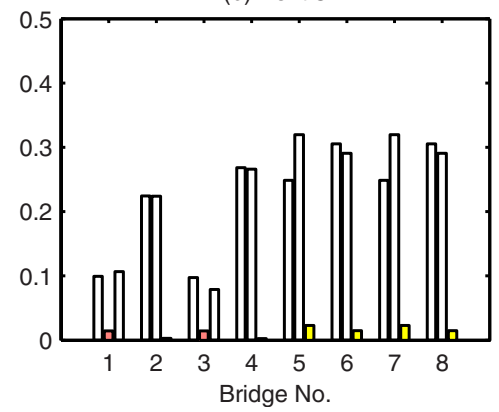

(c) Bent 4

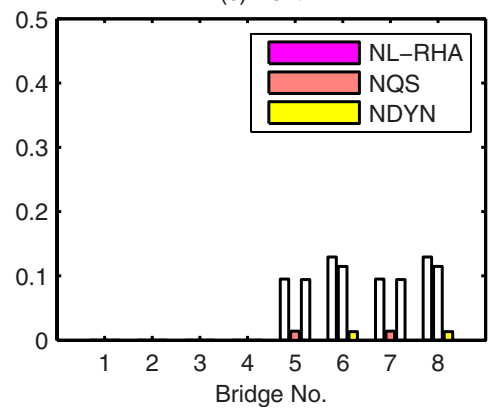

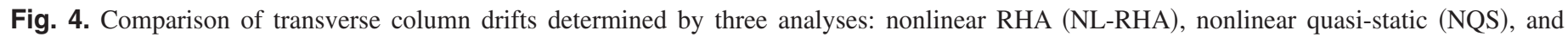
nonlinear dynamic (NDYN). Results are for fault-parallel ground motions associated with a strike-slip fault.

proach demonstrated to be appropriate in a preceding section. Thus, the peak value of the total response is estimated by

$$
r^{t}=r_{o+g}^{s}+r_{o}
$$

where $r_{o+g}^{s}=$ peak value of the quasi-static part of the response (including the effects of gravity loads) and $r_{o}=$ peak value of the dynamic part of the response.

In all three approximate procedures, the peak value of the quasi-static part of the response including the effects of gravity loads, $r_{o+g}^{s}$, is computed by nonlinear static analysis of the bridge due to ground displacements, $\alpha_{l} u_{g o}$, applied simultaneously at all supports, where $u_{g o}=$ peak value of the ground displacement at the reference support. Gravity loads are applied prior to the static analysis and part of the response, $r_{g}$, due to gravity loads is noted.

Presented next are three procedures to estimate the peak value of the dynamic part of the response: modal pushover analysis, linear dynamic analysis, and linear static analysis.

\section{Modal Pushover Analysis}

The modal pushover analysis (MPA) procedure developed earlier for estimating seismic demands for buildings (e.g., Chopra 2007: Sec. 19.7.3) is adapted for bridges crossing fault-rupture zones. The MPA procedure is specialized only for the most-dominant mode because, as demonstrated in the companion paper (Goel and Chopra 2009), only this mode is generally sufficient to accurately estimate the response of many bridges. The procedure is summarized next in step-by-step form:

1. Compute the vibration periods, $T_{n}$, and mode shapes, $\boldsymbol{\phi}_{n}$, of the bridge.

2. Identify the most-dominant mode that needs to be considered in the dynamic analysis based on the modal contribution factors of the linearly elastic bridge as follows:

- 2.1. Compute the effective influence vector, $\mathbf{t}_{\mathrm{eff}}$, as the vector of displacements in the structural DOF obtained by linear static analysis of the bridge due to support displacements $\alpha_{l}$ applied simultaneously as demonstrated in the companion paper; this effective influence vector has no resemblance to the one for spatially uniform excitation.

- 2.2. Compute the response, $r^{\mathrm{st}}$, by static analysis of the bridge due to forces equal to $\mathbf{m}_{\text {eff }}$ applied at the structural DOF.

- 2.3. Compute the modal static response, $r_{n}^{\text {st }}$, by static analysis of the bridge due to forces $\mathbf{s}_{n}=\Gamma_{n} \mathbf{m} \boldsymbol{\phi}_{n}$ applied at the structural DOF, where $\Gamma_{n}=\boldsymbol{\phi}_{n}^{T} \mathbf{m} \mathbf{t}_{\mathrm{eff}} / \boldsymbol{\phi}_{n}^{T} \mathbf{m} \boldsymbol{\phi}_{n}$.

- 2.4. Compute the modal contribution factor for the $n$th mode, $\bar{r}_{n}=r_{n}^{\text {st }} / r^{\text {st }}$ (Chopra 2007: Sec. 12.10).
- 2.5. Repeat Steps 2.3 and 2.4 for all modes.

- 2.6. Select the most-dominant mode as the mode with the largest modal contribution factor.

3. Compute the peak value of dynamic response, $r_{n 0}$, in the most-dominant mode of the bridge by nonlinear static (or pushover) analysis as follows:

- 3.1. Develop the pushover curve, $\beta_{n}-u_{r n}$, for the modal force distribution, $\mathbf{f}_{n}^{*}=\beta_{n} \mathbf{m} \boldsymbol{\phi}_{n}$, in which $\beta_{n}$ is the forcescale factor, and $u_{r n}$ is the displacement of the bridge at a reference point. Gravity loads are applied before pushover analysis and $P-\Delta$ effects are included. Note the value of the reference point displacement, $u_{r g}$, and the value of the desired dynamic response, $r_{g}$, due to gravity loads.

- 3.2. Convert the $\beta_{n}-u_{r n}$ pushover curve to the forcedisplacement, $F_{s n} / L_{n}-D_{n}$, relation for the inelastic single degree-of-freedom (SDF) system by utilizing $F_{s n} / L_{n}$ $=\beta_{n} / \Gamma_{n}$ and $D_{n}=u_{r n} / \Gamma_{n} \phi_{r n}$ in which $\phi_{r n}=$ value of $\boldsymbol{\phi}_{n}$ at the reference point; these relations are developed in the Appendix.

- 3.3. Idealize the pushover curve, as necessary, and define appropriate hysteretic rules for cyclic deformations.

- 3.4. Compute the peak deformation $D_{n}$ of the inelastic SDF system defined by the force-deformation relation developed in Step 3.3 and damping ratio $\zeta_{n}$, subjected to the ground acceleration $\ddot{u}_{g}(t)$ at the reference support.

- 3.5. Calculate peak value of the reference-point displacement $u_{r n}$ from $u_{r n}=\Gamma_{n} \phi_{r n} D_{n}$.

- 3.6. At the reference point displacement equal to $u_{r g}+u_{r n}$, note the value $r_{n+g}$ of desired response from the pushover data.

4. The peak dynamic response is, $r_{o}=r_{n+g}-r_{g}$, where $r_{g}$ $=$ contribution of gravity loads alone, computed earlier in Step 3.1.

Note that the most-dominant mode to be considered in the dynamic analysis may depend on the response quantity under consideration, e.g., most-dominant mode for computation of the dynamic part of the drift at one bent may differ from that for another bent. Therefore, the MPA procedure must be implemented for each mode that is identified to be the most-dominant mode for a seismic response of interest, implying the need for several such analyses. For bridges considered in this investigation, however, it was found that generally the same mode was the most-dominant mode for all seismic responses [see modal contribution factors in Tables 1-4 of the companion paper Goel and Chopra (2009)], requiring a single implementation of the MPA procedure. Only in very few cases [such as the example bridge in Goel and Chopra (2008a)] did the most-dominant mode differ for different seismic 


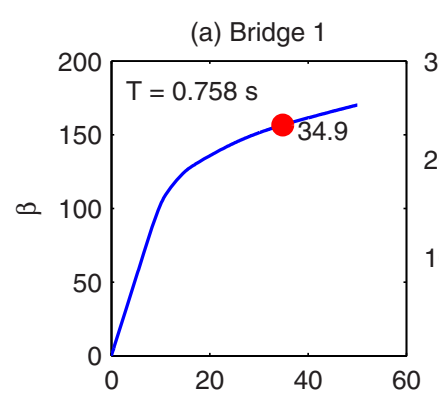

(b) Bridge 2

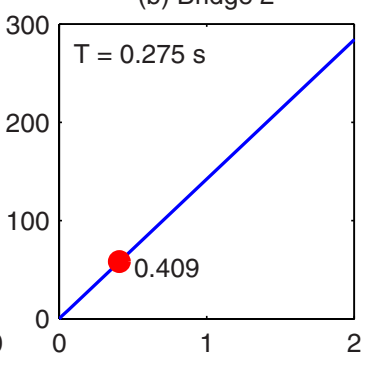

(e) Bridge 5
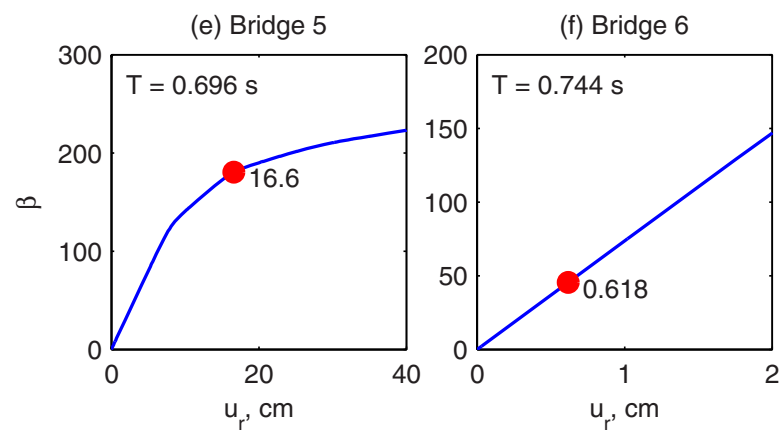

(c) Bridge 3

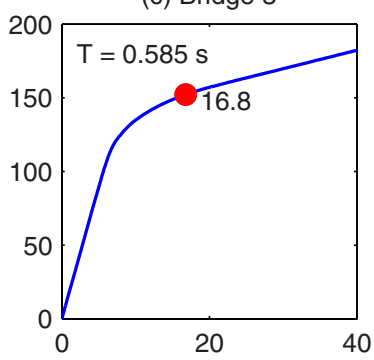

(g) Bridge 7

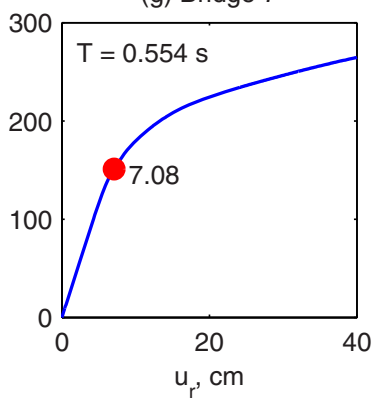

(d) Bridge 4

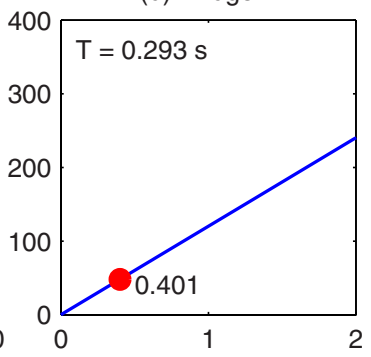

(h) Bridge 8

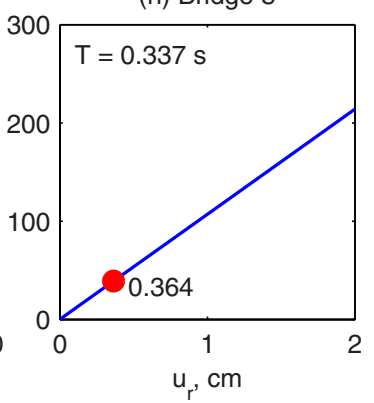

Fig. 5. Pushover curve and peak reference displacement for the most-dominant mode of the selected bridges

responses. Even for such cases, implementation of the MPA procedure was needed for no more that two different modes.

\section{Pushover Curve and Reference-Point Displacement}

Fig. 5 presents the pushover curves for the most-dominant mode - the one for drift in Bent 2-of the eight selected bridges, together with the peak value of the transverse displacement at Abutment 1, chosen as the reference displacement. Bridges with elastic shear keys, e.g., Bridges 2, 4, 6, and 8, remain within the linear-elastic range during the dynamic part of the response (Fig. 5) because the peak displacement is very small (Figs. 3 and 4). Bridges without shear keys, e.g., Bridges 1, 3, 5, and 7, on the other hand, are deformed beyond the elastic limit, but only slightly (Fig. 5).

These results suggest that linear analysis may be adequate to estimate the dynamic part of the response of these bridges. To evaluate this approximation, the peak reference displacements determined by nonlinear and linear analyses are compared in Fig. 6 for bridges without shear keys. This comparison shows that linear analysis slightly underestimates the reference displacement for

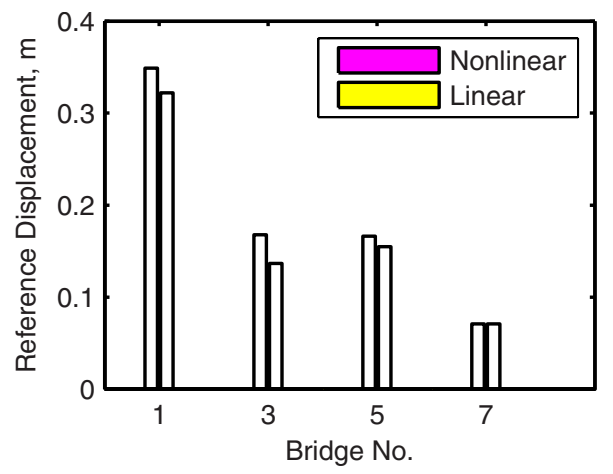

Fig. 6. Comparison of the reference displacement for the mostdominant mode from nonlinear and linear analyses
Bridges 1, 3, and 5 but provides an excellent estimate for Bridge 7; however, the slight underestimation of the peak displacement appears to be well within the errors acceptable for most practical applications. For practical implementation of linear analysis of the dynamic part of the response, two simple procedures are presented next.

\section{Linear Dynamic Analysis}

The peak modal response of a structure due to one mode- the most-dominant mode-can be determined by linear analysis of the bridge due to equivalent static forces (see Chopra 2007, Sec. 13.1)

$$
\mathbf{f}_{n}=\mathbf{s}_{n} A_{n}=\Gamma_{n} \mathbf{m} \phi_{n} A_{n}
$$

in which $\Gamma_{n}$ was defined in Step 2.3 of the MPA procedure, and $A_{n}$ is determined from the pseudo-acceleration spectrum for the reference support acceleration $\ddot{u}_{g}(t)$. As will be demonstrated later, this spectrum differs significantly from the CALTRANS SDC spectrum. The linear dynamic analysis procedure is equivalent to the RSA procedure in the companion paper (Goel and Chopra 2009), but specialized to consider only one mode-the mostdominant mode.

The peak value, $r_{o}$, of the dynamic part of the response can be computed as follows:

1. Compute the vibration periods, $T_{n}$, and mode shapes, $\boldsymbol{\phi}_{n}$, of the bridge.

2. Compute the effective influence vector, $\mathbf{\iota}_{\text {eff }}$, as the vector of displacements in the structural DOF obtained by linear static analysis of the bridge due to support displacements $\alpha_{l}$ applied simultaneously in the appropriate direction: faultparallel or normal-fault.

3. Identify the most-dominant mode by implementing Step 2.2 of the MPA procedure presented earlier and compute $\Gamma_{n}$ $=\boldsymbol{\phi}_{n}^{T} \mathbf{m}_{\mathrm{eff}} / \boldsymbol{\phi}_{n}^{T} \mathbf{m} \boldsymbol{\phi}_{n}$.

4. Estimate $r_{o}$ by linear analysis of the bridge due to equivalent static forces $s_{n}=\Gamma_{n} \mathbf{m} \phi_{n} A_{n}$. 

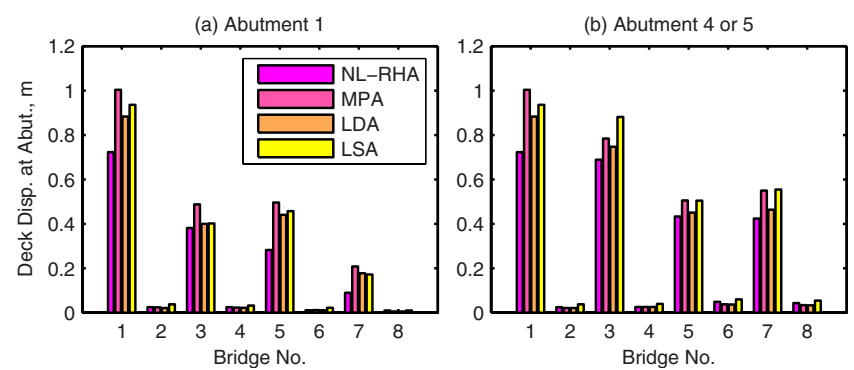

Fig. 7. Transverse deck displacement at abutment determined by three approximate procedures-MPA, linear dynamic analysis (LDA), and linear static analysis (LSA) — and exact nonlinear RHA (NL-RHA). Results are for fault-parallel ground motions associated with a vertical strike-slip fault.

\section{Linear Static Analysis}

As demonstrated in the companion paper (Goel and Chopra 2009), the peak value of the dynamic part of the response of linearly elastic bridges can be estimated to a sufficient accuracy simply by static analysis of the structure due to lateral force $=2.5 \mathrm{~m}_{\text {eff }} \ddot{u}_{g o}$; computation of vibration periods and modes is no longer necessary. The same procedure is adopted for inelastic bridges because, as demonstrated earlier, the dynamic part of their response may be estimated by linear analysis.

Thus, the peak value $r_{o}$ of the dynamic part of the response can be computed as follows:

1. Compute the effective influence vector, $\mathbf{t}_{\mathrm{eff}}$, as the vector of displacements in the structural DOF obtained by linear static analysis of the bridge due to support displacements $\alpha_{l}$ applied simultaneously.

2. Estimate $r_{o}$ by linear static analysis of the bridge due to lateral force $=2.5 \mathbf{m i}_{\text {eff }} \ddot{u}_{g o}$.

\section{Accuracy of Approximate Procedures}

The total (quasi-static plus dynamic) seismic demands for bridges oriented orthogonal to strike-slip faults due to fault-parallel ground motions estimated by the three approximate procedures are compared against results of exact nonlinear RHA. Recall that the three procedures are identical in their computation of the quasi-static response but differ in estimation of the dynamic response. The results presented in Figs. 7 and 8 lead to the follow-
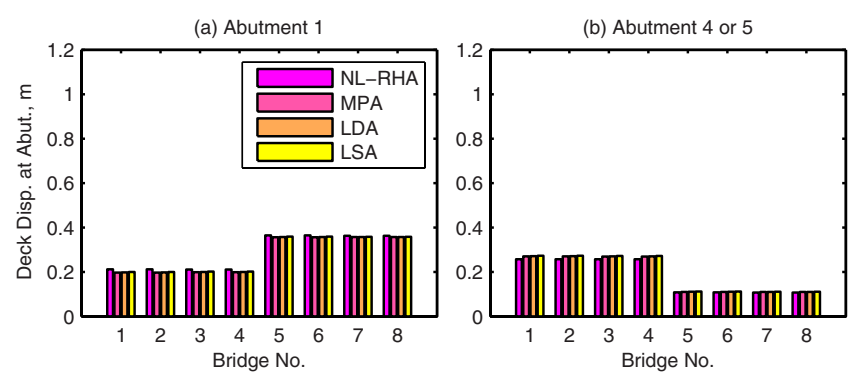

Fig. 9. Longitudinal deck displacement at abutment determined by three approximate procedures-MPA, linear dynamic analysis (LDA), and linear static analysis (LSA) — and exact nonlinear RHA (NL-RHA). Results are for fault-normal ground motions associated with a fault with dip of $40^{\circ}$ and rake of $110^{\circ}$.

ing observations:

First, the MPA procedure leads to estimates of deck displacements at abutments and column drifts that are generally very close to those from the nonlinear RHA. The exception occurs for Bridges 5 and 7 where the MPA procedure underestimates the columns drift in Bent 2 [Fig. 8(a)]. This appears to be the case because modes other than the most-dominant mode contribute noticeably to the dynamic part of the total column drift. Second, the simpler linear dynamic analysis procedure is generally no less accurate than the computationally more demanding MPA procedure. Third, the linear static procedure, which is the simplest of the three approximate procedures, generally provides estimates of deck displacements at abutments (Fig. 7) and column drifts (Fig. 8) that are also very close to those from the nonlinear RHA. In general, accuracy of the linear static procedure is not much worse than that of the MPA procedure. Note that the linear static procedure underestimates the drift in Bent 2 of three bridges without shear keys-Bridges 3, 5, and 7-when compared to the MPA procedure [Fig 8(a)]. However, as would be demonstrated later in this paper, the upper bound of the drift in Bent 2 of these bridges is controlled by the bridges with elastic shear keys-Bridges 4, 6, and 8 - for which accuracy of the linear static procedure is similar to that of the MPA procedure. Therefore, linear static analysis is preferable over linear dynamic analysis or MPA for practical applications to ordinary bridges.

The results presented in Figs. 9 and 10 for fault-normal motions on a fault with dip of $40^{\circ}$ and rake of $110^{\circ}$ indicate that all three procedures-MPA, linear dynamic analysis, and linear static (a) Bent 2

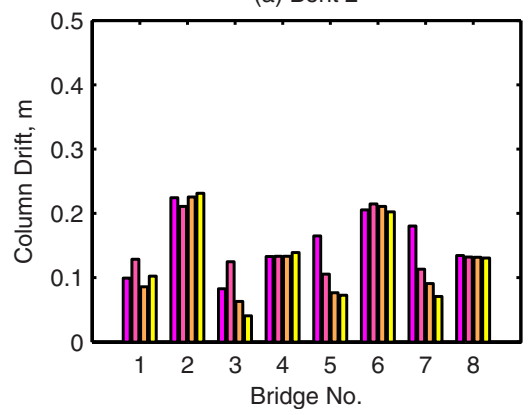

(b) Bent 3

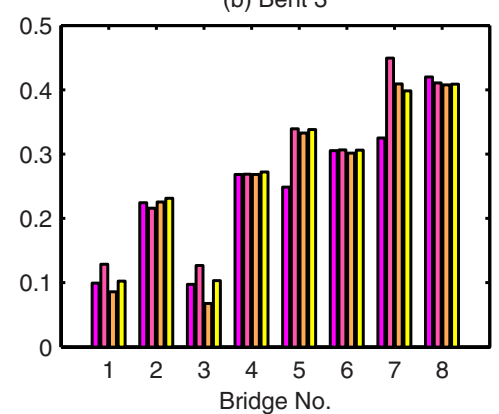

(c) Bent 4

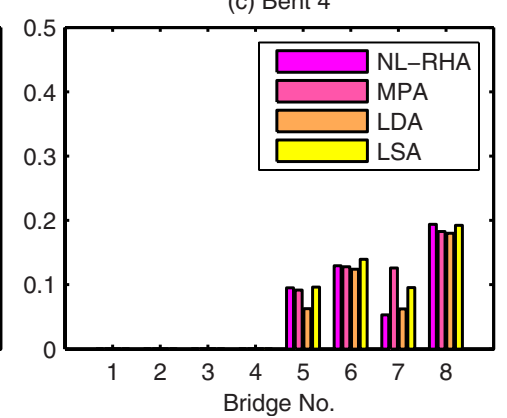

Fig. 8. Transverse column drifts at abutment determined by three approximate procedures-MPA, linear dynamic analysis (LDA), and linear static analysis (LSA) — and exact nonlinear RHA (NL-RHA). Results are for fault-parallel ground motions associated with a vertical strike-slip fault. 
(a) Bent 2

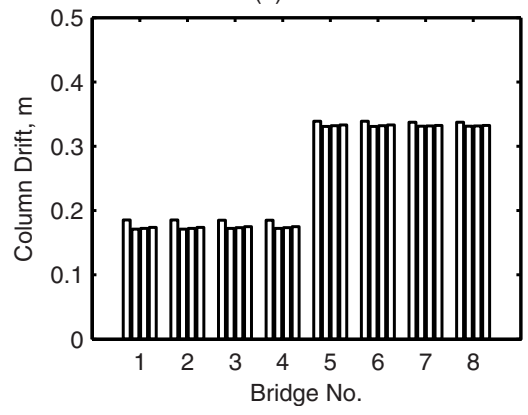

(b) Bent 3

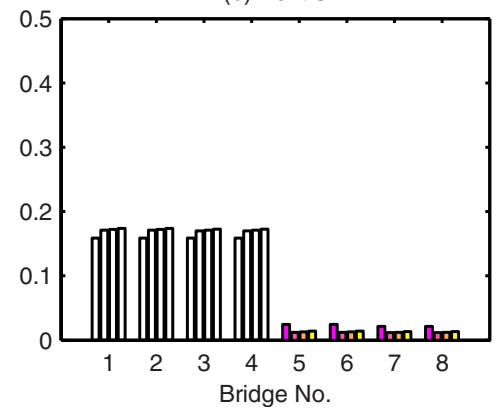

(c) Bent 4

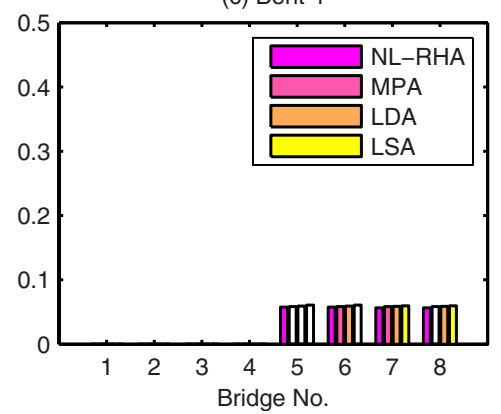

Fig. 10. Longitudinal column drifts at abutment determined by three approximate procedures-MPA, linear dynamic analysis (LDA), and linear static analysis (LSA) — and exact nonlinear RHA (NL-RHA). Results are for fault-normal ground motions associated with a fault with dip of $40^{\circ}$ and rake of $110^{\circ}$.

analysis - provide estimates that are essentially identical, and are very close to those from the exact nonlinear RHA. Thus, as before, the simpler linear static analysis is preferable over linear dynamic analysis or MPA for practical applications. In passing, observe that the longitudinal response of a bridge oriented orthogonal to the fault is not affected by shear keys (compare Bridges 1 and 2, 3 and 4, 5 and 6, and 7 and 8 in Figs. 9 and 10), because they provide restraint only in the transverse direction.

\section{Application to Bridges with Nonlinear Shear Keys}

A recent paper (Goel and Chopra 2008b) demonstrated that the earthquake response of bridges crossing fault-rupture zones is very sensitive to the strength of the shear keys. Computations of this response were shown to be unreliable for lack of experimental data and realistic nonlinear force-deformation models for shear keys. For this reason, it was proposed to estimate bridge response as the larger of responses computed by nonlinear analysis of the bridge for two shear key cases: no shear keys and elastic shear keys. Therefore, this upper bound of response is selected as the benchmark to evaluate approximate procedures presented in this paper.

Figs. 11-14 present the upper bound response of the four bridges considered in this investigation: three-span symmetric (3S), three-span unsymmetric (3U), four-span symmetric (4S), and four-span unsymmetric $(4 \mathrm{U})$ determined by the three approximate procedures-MPA, linear dynamic analysis, and linear static analysis - and nonlinear RHA. Included are results for transverse
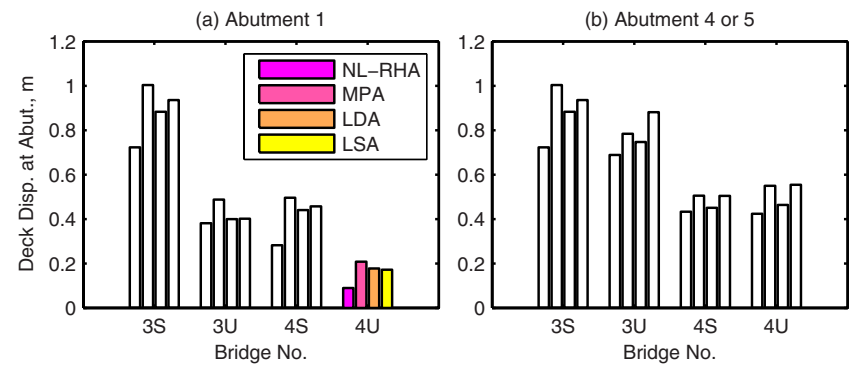

Fig. 11. Upper bound of transverse deck displacement at abutment determined by three approximate procedures-MPA, linear dynamic analysis (LDA), and linear static analysis (LSA) — and exact nonlinear RHA (NL-RHA). Results are for fault-parallel ground motions associated with a vertical strike-slip fault. response due to fault-parallel ground motions on a strike-slip fault (Figs. 11 and 12) and for longitudinal response due to faultnormal motions on a fault with a dip of $40^{\circ}$ and rake of $110^{\circ}$ (Figs. 13 and 14).

The MPA procedure provides a conservative estimate of deck displacements at abutments. They are within about $10 \%$ of the result from nonlinear RHA for a few cases [Bridges $3 \mathrm{U}$ and $4 \mathrm{~S}$ in Fig. 11(a)]. For most of the remaining cases, the results from the MPA procedure are within about $30 \%$ of the estimate from nonlinear RHA [see Bridge $3 \mathrm{~S}$ and $3 \mathrm{U}$ in Fig. 11(a) and bridges $3 \mathrm{~S}$ and $4 \mathrm{U}$ in Fig. 11(b)]. The apparently much larger percentage discrepancy [Bridge $4 \mathrm{U}$ in Fig. 11(a)] is inconsequential as the response under consideration is very small. The MPA procedure provides estimates of column drifts that are generally very close to the exact results (Fig. 12), within about 5\% for most cases [Bridges 3S, 3U, and 4S in Fig 12(a); Bridges 3S, 3U, and $4 \mathrm{U}$ in Fig. 12(b); and Bridges $4 \mathrm{~S}$ and $4 \mathrm{U}$ in Fig 12(c)], within $10 \%$ for one case [Bridge $4 \mathrm{~S}$ in Fig. 12(b)], and about $30 \%$ for another case [Bridge 4U in Fig 12(a)]. Although the MPA overestimated the deck displacements at abutments, it slightly underestimated the column drifts for a few cases [Bridges $3 \mathrm{~S}$ and $4 \mathrm{U}$ in Fig. 12(a); Bridge $3 \mathrm{~S}$ in Fig. 12(b); and Bridge $4 \mathrm{U}$ in Fig. 12(c)].

The linear dynamic analysis and linear static analysis procedures also provide conservative estimates of deck displacements at abutments [Fig. 11(a)], but these procedures are generally slightly less conservative compared to the MPA procedure. The exception occurs for Bridge $3 \mathrm{U}$ for which the linear static analysis procedure provides slightly more conservative estimate of deck displacements at abutments (Fig. 11). The columns drifts estimated by linear dynamic analysis and linear static analysis procedures are generally very similar to those from the MPA procedure (Fig. 12).

The results presented in Figs. 13 and 14 for longitudinal response due to fault-normal motions on a fault with a dip of $40^{\circ}$ and rake of $110^{\circ}$ indicate that the three approximate proceduresMPA, linear dynamic analysis, and linear static analysis-provide essentially identical estimates of deck displacements at abutments and column drifts, which are within about $5 \%$ of the exact results.

The three approximate procedures are much more accurate in estimating the upper bound of the response for the two shear-key cases (Figs. 11-14) compared to that observed previously for the individual cases (see Figs. 7-10). The accuracy of the linear static analysis procedure is generally no worse, and slightly better for many cases, compared to the MPA procedure or the linear dynamic analysis procedure. Therefore, the linear static analysis procedure, which is the simplest of the three approximate proce- 
(a) Bent 2

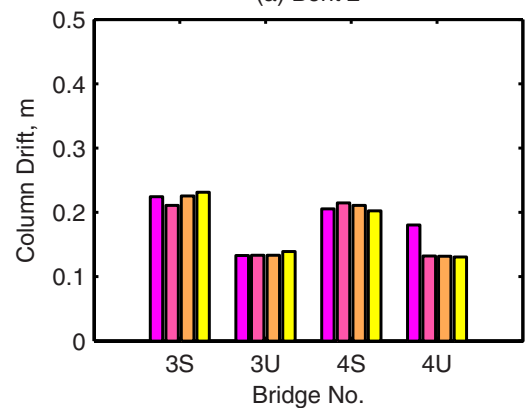

(b) Bent 3

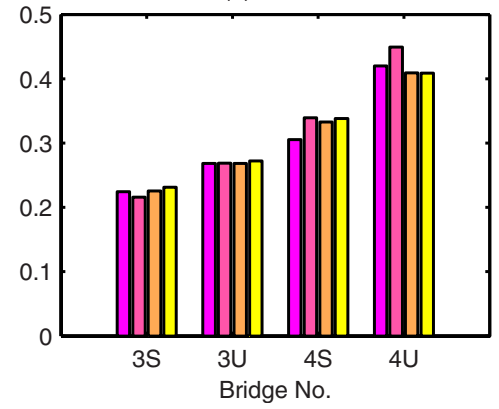

(c) Bent 4

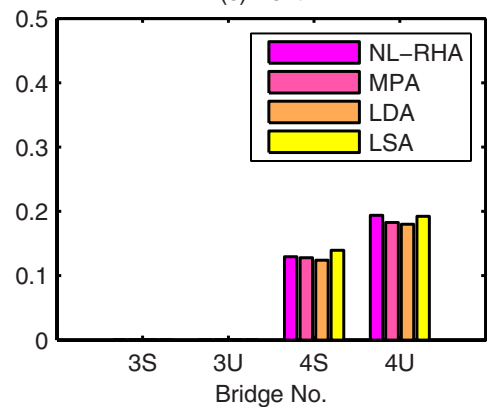

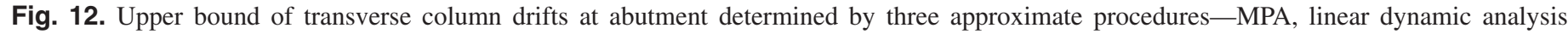

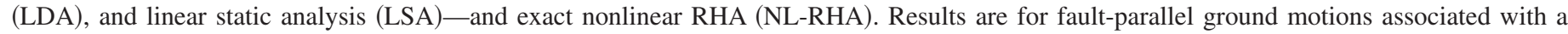
vertical strike-slip fault.

dures, is preferable over MPA or linear dynamic analysis procedures.

\section{Comments on Current Procedure}

Recognizing the difficulty in implementing nonlinear RHA with spatially varying ground motions, practicing engineers have devised a simple two-step procedure for design of bridges crossing fault-rupture zones (CALTRANS, personal communication, 2007). The first step in this procedure estimates the displacement demands for the bridge, assumed to be linearly elastic by standard RSA. Implicit in this approach is the assumption that the bridge is located on one side of the fault and thus subjected to spatially uniform excitation. The excitation is characterized either by a site-specific spectrum or the CALTRANS SDC spectrum that is modified for near-field effects; the magnification factor is zero for $T \leqslant 0.5 \mathrm{~s}, 20 \%$ for $T \geqslant 1 \mathrm{~s}$, and varies linearly over the period range $0.5<T<1 \mathrm{~s}$ (CALTRANS 2006). The second step estimates the displacement capacity of the bridge by nonlinear static analysis wherein gravity loads are applied first, followed by faultrupture displacements applied at various supports of the bridge. One-half of the fault-rupture displacement is applied to the portion of the bridge on one side of the fault; the other one-half is applied in the opposite direction to the portion of the bridge on the other side of the fault. This part of analysis in the second step may be interpreted as equivalent to the quasi-static analysis described in preceding sections. Finally, in the second step, lateral forces proportional to the structural mass distribution are applied
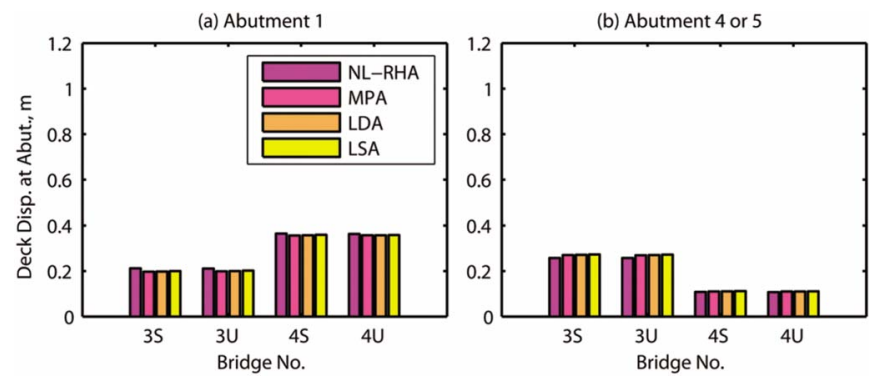

Fig. 13. Upper bound of longitudinal deck displacement at abutment determined by three approximate procedures-MPA, linear dynamic analysis (LDA), and linear static analysis (LSA procedures) - and exact nonlinear RHA (NL-RHA). Results are for fault-normal ground motions associated with a fault with dip of $40^{\circ}$ and rake of $110^{\circ}$. to the bridge and monotonically increased until column plastic hinges reach their capacity and incremental displacement is noted. The bridge design is acceptable if the incremental displacement capacity determined by this pushover analysis exceeds the seismic displacement demand from RSA in the first step. The last step of the procedure suggests that the total seismic demand is estimated by superposition of nonlinear quasi-static response due to peak values of support displacements, and linear dynamic response of the bridge, assumed to be located one side of the fault, due to spatially uniform support excitation.

Although combining quasi-static and dynamic responses in the above-described simplistic procedure appears to be similar to the superposition approach in the three approximate procedures, there are two important discrepancies in computation of the dynamic part of the response. The first step of the simplistic procedure assumes the bridge to be located on one side of the fault and thus subjected to spatially uniform support excitation, which bears no resemblance to spatially varying excitation with fault offset relevant for bridges crossing fault-rupture zones. The same assumption is implicit in the final part of the second step in the simplistic procedure. The mass-proportional lateral force distribution, which is equivalent to $\mathbf{s}^{*}=\mathbf{m} \mathbf{t}$, used in the pushover analysis of the simplistic procedure, may be appropriate for bridges located on one side of the fault that are subjected to spatially uniform support excitation, but not for bridges crossing fault-rupture zones subjected to spatially varying support excitation with fault offset. For bridges crossing fault-rupture zones, the appropriate force distribution is either that corresponding to the most-dominant mode, i.e., $\mathbf{s}_{n}^{*}=\mathbf{m} \boldsymbol{\phi}_{n}$, or that considering the distribution of inertia forces on the bridge subjected to spatially varying support motions with fault offset, i.e., $\mathbf{s}^{*}=\mathbf{m}_{\text {eff. }}$. As demonstrated in the companion paper (Goel and Chopra 2009), the influence vector, ו, for spatially uniform support excitation has no resemblance to the effective influence vector, $\mathbf{l}_{\text {eff }}$, for spatially varying excitation with fault offset.

Second, the response spectrum used in the simplistic procedure is inappropriate for ground motions expected in close proximity to faults. This becomes apparent by comparing the CALTRANS SDC spectrum with the response spectrum for ground motions in fault-rupture zones, all presented in normalized form [see Fig. 7 of companion paper (Goel and Chopra 2009)].

In contrast, all three approximate procedures, MPA, linear dynamic analysis, and linear static analysis, recognize all the important features of the earthquake response of bridges crossing faultrupture zones: spatial variations including fault offset in the support motions, and the characteristics of ground motions ex- 
(a) Bent 2

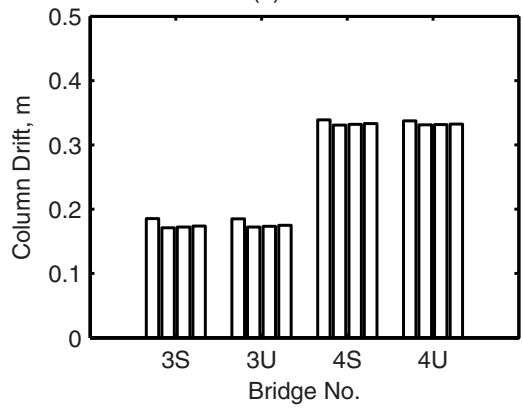

(b) Bent 3

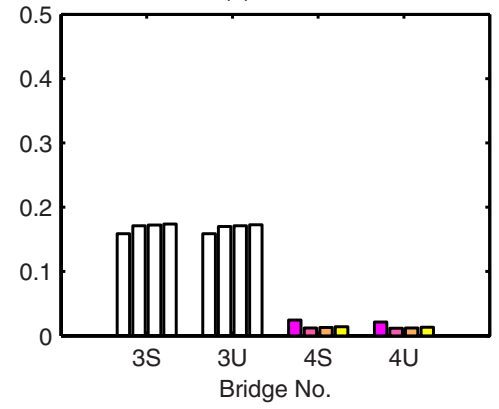

(c) Bent 4

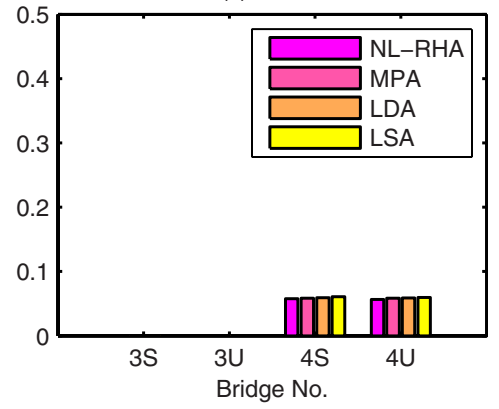

Fig. 14. Upper bound of longitudinal column drifts at abutment determined by three approximate procedures-MPA, linear dynamic analysis (LDA), and linear static analysis (LSA procedures) — and exact nonlinear RHA (NL-RHA). Results are for fault-normal ground motions associated with a fault with dip of $40^{\circ}$ and rake of $110^{\circ}$.

pected in close proximity to the causative fault. Linear static analysis, the simplest of the three procedures presented here, is especially attractive for practical application because it is even simpler than the simplistic procedure, and yet provides good estimates of seismic demands, because it is rooted in the structural dynamics theory.

\section{Conclusions and Recommendation}

The seismic demands for ordinary bridges crossing fault-rupture zones can be estimated to a useful degree of accuracy by superposition of the peak values of the quasi-static and dynamic parts of the response [Eq. (2)]. The peak value of the quasi-static part of the response, including the effects of gravity loads, is computed by nonlinear static analysis of the bridge due to peak ground displacements applied simultaneously at all supports.

Three approximate procedures were presented for estimating the peak value of the dynamic part of the response. The linear static analysis procedure, which is simpler than the two other procedures, MPA and linear dynamic analysis, is recommended as the procedure for practical analysis of ordinary bridges. Although the other two are dynamics-based procedures, they consider only the response contribution of the most-dominant mode; at the expense of additional computational effort, they can be extended to include higher mode contributions; as in the general MPA (Chopra 2007) and RSA (Goel and Chopra 2009) procedures, respectively. On the other hand, the linear static analysis procedure does not require computation of the vibration periods or modes of the structure, but indirectly considers contributions of all vibration modes and requires only a linear static analysis of the bridge due to lateral forces that recognize the ground offset across the fault and the shape of the response spectrum for fault ground motions in close proximity to the fault.

\section{Acknowledgments}

The research reported in this paper is supported by CALTRANS under Contract No. 59A0435 with Mahmoud Khojasteh as the project manager. This support is gratefully acknowledged. Also acknowledged is the assistance of Douglas Dreger and Gabriel Hurtado of University of California, Berkeley who generated the ground motions used in this investigation.

\section{Appendix. Properties of Inelastic SDF System}

The MPA procedure considering lateral force distribution corresponding to only one mode - the most-dominant mode-is based on the assumption that response of a nonlinear MDF system occurs due to that mode alone. This implies that response due to other modes and the coupling between modes due to system nonlinearity is ignored. For such an assumption, Eq. (1) for dynamic response of a nonlinear MDF system may be rewritten as

$$
\mathbf{m} \ddot{\mathbf{u}}+\mathbf{c} \dot{\mathbf{u}}+\mathbf{f}_{s}(\mathbf{u}, \dot{\mathbf{u}})=-\mathbf{s}_{n} \ddot{u}_{g}(t)
$$

in which $s_{n}=\Gamma_{n} \mathbf{m} \boldsymbol{\phi}_{n}, \Gamma_{n}=\phi_{n}^{T} \mathbf{m}_{\mathrm{eff}} / \boldsymbol{\phi}_{n}^{T} \mathbf{m} \boldsymbol{\phi}_{n}$, and $n=$ number of the most-dominant mode. Premultiplying Eq. (4) by $\boldsymbol{\phi}_{n}^{T}$ and using the mass- and classical damping-orthogonality property of modes gives

$$
\ddot{q}_{n}+2 \zeta_{n} \omega_{n} \dot{q}_{n}+\frac{F_{s n}}{M_{n}}=-\Gamma_{n} \ddot{u}_{g}(t)
$$

where $F_{s n}=\boldsymbol{\phi}_{n}^{T} \mathbf{f}_{s}(\mathbf{u}, \dot{\mathbf{u}})$ and $M_{n}=\boldsymbol{\phi}_{n}^{T} \mathbf{m} \boldsymbol{\phi}_{n}$. The response of the MDF nonlinear system then can be computed from

$$
\mathbf{u}=\mathbf{u}_{n}=\boldsymbol{\phi}_{n} q_{n}=\Gamma_{n} \boldsymbol{\phi}_{n} D_{n}
$$

and displacement at any reference location from

$$
u_{r n}=\Gamma_{n} \phi_{r n} D_{n}
$$

in which $D_{n}$ is governed by

$$
\ddot{D}_{n}+2 \zeta_{n} \omega_{n} \dot{D}_{n}+\frac{F_{s n}}{L_{n}}=-\ddot{u}_{g}(t)
$$

with $L_{n}=\boldsymbol{\phi}_{n}^{T} \mathbf{m}_{\text {eff. }}$. Note that Eq. (8) is the governing equation of motion of an inelastic SDF system with $\omega_{n}$ (or $\left.T_{n}\right), \zeta_{n}$, and forcedeformation behavior defined by the $F_{s n} / L_{n}$ relationship subjected to ground motion at a reference support. Utilizing Eqs. (7) and (8), the force-deformation relationship of the inelastic SDF system needed in the MPA procedure can be obtained from that of the MDF system from

$$
\begin{aligned}
& \frac{F_{s n}}{L_{n}}=\frac{\boldsymbol{\phi}_{n}^{T} \mathbf{f}_{s}}{L_{n}} \\
& D_{n}=\frac{u_{r n}}{\Gamma_{n} \phi_{r n}}
\end{aligned}
$$


The pushover analysis for the most-dominant mode involves applying increasing intensity of the force distribution given by

$$
\mathbf{f}_{s}=\beta_{n} \mathbf{m} \phi_{n}
$$

in which $\beta_{n}$ is the force-scale factor during pushover analysis. Utilizing Eq. (10) into Eq. (9a) gives

$$
\frac{F_{s n}}{L_{n}}=\frac{\boldsymbol{\phi}_{n}^{T} \mathbf{f}_{s}}{L_{n}}=\frac{\beta_{n} \boldsymbol{\phi}_{n}^{T} \mathbf{m} \boldsymbol{\phi}_{n}}{L_{n}}=\frac{\beta_{n} M_{n}}{L_{n}}=\frac{\beta_{n}}{\Gamma_{n}}
$$

Therefore, the pushover curve for a MDF system can be converted to the $F_{s n} / L_{n}-D_{n}$ curve of the inelastic SDF system by Eqs. (11) and (9b).

Although not essential, the $F_{s n} / L_{n}-D_{n}$ relation is often idealized as a bilinear (or multilinear) curve because most readily available computer programs for solving response of inelastic SDF system utilize such force-deformational idealization. The initial slope of this curve is equal to $\omega_{n}^{2}$ indicating that the vibration period $T_{n}$ of the inelastic SDF system is given by

$$
T_{n}=2 \pi\left(\frac{L_{n} D_{n y}}{F_{\text {sny }}}\right)^{1 / 2}
$$

in which subscript $y$ indicates the yield values. This value of $T_{n}$, which may differ from the period of the corresponding linear system, should be used for estimating deformation of the inelastic SDF system.

The pushover curves for a multistory building is plot of base shear, $V_{b n}$, versus roof displacement, $u_{r n}$. From such a pushover curve, $F_{s n} / L_{n}$ for the $n$th mode inelastic SDF systems is computed from

$$
\frac{F_{s n}}{L_{n}}=\frac{V_{b n}}{M_{n}^{*}}
$$

in which $M_{n}^{*}=L_{n} \Gamma_{n}$ is the effective modal mass. The relationship of Eq. (13) for a multistory building is a special case of Eq. (11), which becomes evident from the following equations:

$$
V_{b n}=\mathbf{1}^{T} \mathbf{f}_{s n}=\beta_{n} \mathbf{1}^{T} \mathbf{m} \boldsymbol{\phi}_{n}=\beta_{n} \boldsymbol{\phi}_{n}^{T} \mathbf{m} \mathbf{1}=\beta_{n} L_{n}
$$

$$
\frac{V_{b n}}{M_{n}^{*}}=\frac{V_{b n}}{\Gamma_{n} L_{n}}=\frac{\beta_{n} L_{n}}{\Gamma_{n} L_{n}}=\frac{\beta_{n}}{\Gamma_{n}}
$$

which utilize the fact that $\mathbf{t}_{\mathrm{eff}}=\mathbf{l}=\mathbf{1}$ for buildings subjected to spatially-uniform support excitation.

Although the $V_{b n}-u_{r n}$ pushover curve is useful for design and evaluation of buildings, where it can provide useful insight into nonlinear behavior and potential weak spots of the selected building, it may not be appropriate for bridges crossing fault-rupture zones because the most-dominant mode, which often involves torsional motions about a vertical axis, may induce little or no base shear. The value of $\beta_{n}$ would always be non-zero during pushover analysis using force distributions for all types of modes. Therefore, the $\beta_{n}-u_{r n}$ pushover curve is more appropriate for bridges crossing fault-rupture zones.

\section{References}

CALTRANS. (2006). Seismic design criteria, Version 1.4.

Chopra, A. K. (2007). Dynamic of structures: Theory and applications to earthquake engineering, 3rd Ed., Prentice-Hall, Englewood Cliffs, N.J.

Goel, R. K., and Chopra, A. K. (2008a). "Analysis of ordinary bridges crossing fault-rupture zones." Rep. No. UCB/EERC-2008/01, Earthquake Engineering Research Center, Univ. of California, Berkeley, Calif.

Goel, R. K., and Chopra, A. K. (2008b). "Role of shear keys in seismic behavior of bridges crossing fault-rupture zones." J. Bridge Eng., 13(4), 398-408.

Goel, R. K., and Chopra, A. K. (2009). "Linear analysis of ordinary bridges crossing fault-rupture zones." J. Bridge Eng., 14(3), 203-215.

Mander, J. B., Priestly, M. J. N., and Park, R. (1988). "Theoretical stressstrain model for confined concrete." J. Struct. Eng., 114(8), 18041826.

McKenna, F., and Fenves, G. (2001). The OpenSees command language manual: Version 1.2, Pacific Earthquake Engineering Center, Univ. of California, Berkeley, Calif., 〈http://opensees.berkeley.edu〉. 